\title{
The effect of nucleos(t)ide analogues on clinical outcomes of patients treated with transarterial chemoembolization and radiofrequency abla- tion for hepatitis B virus-related hepatocellular carcinoma
}

\author{
Jae Min Park, Won Hyeok Choe, Jeong Han Kim, So Young Kwon, Byung Chul Yoo \\ Department of Internal Medicine, Konkuk University Medical Center, Konkuk University School of Medicine, Seoul, Korea
}

Received Aug. 24, 2021

Revised Sep. 13, 2021

Accepted Sep. 22, 2021
Background/Aims: Because hepatitis B virus (HBV) replication has been known to play an important role in cancer recurrence after curative treatment of HBV-related hepatocellular carcinoma (HCC), we examined whether treatment based on nucleos(t)ide analogues (NAs) might decrease the recurrence rate and improve patient survival.

Methods: The retrospective cohort study enrolled 73 patients with chronic hepatitis B who were treated with transarterial chemoembolization (TACE) and radiofrequency ablation (RFA) with curative intent for HCC. Among those, 30 and 43 patients were treated with tenofovir disoproxil fumarate (TDF) and entecavir (ETV), respectively.

Results: Of the 73 patients, 51 experienced HCC recurrence, and 14 patients were dead during a follow-up of $73 \pm 34$ months. Multivariate analyses showed that tumor size (hazard ratio $[\mathrm{HR}], 1.590 ; 95 \%$ confidence-interval $[\mathrm{Cl}], 1.106-2.285 ; P=0.012$ ) and Child-Pugh class $B$ (vs. class $\mathrm{A} /$ non cirrhosis; $\mathrm{HR}, 5.794 ; 95 \% \mathrm{Cl}, 2.311-14.523 ; P=0.001$ ) was significantly associated with $\mathrm{HCC}$ recurrence, and Child-Pugh class $\mathrm{B}(\mathrm{HR}, 7.357 ; 95 \% \mathrm{Cl}, 2.100-25.777 ; P=0.002)$ was an independent unfavorable prognostic factor for survival. During NAs therapy, TDF was superior to ETV for complete viral response at 1 year after the date of combination of TACE and RFA $(P=0.016)$. However, the risks of HCC recurrence and survival were not significantly different between those treated with TDF versus ETV.

Conclusions: TDF was superior to ETV for achieving complete viral response. However, the recurrence and mortality after TACE and RFA for HBV-related HCC were not significantly different between patients treated with TDF versus ETV. (J Liver Cancer 2021;21:155-162)

Keywords: Hepatitis B; Hepatocellular carcinoma; Nucleos(t)ide analogues; Radiofrequency ablation; Transarterial chemoembolization

\section{INTRODUCTION}

Hepatitis B virus (HBV) infection is a major cause of

\author{
Corresponding author: Won Hyeok Choe \\ Department of Internal Medicine, Konkuk University Medical Center, \\ Konkuk University School of Medicine, 120-1 Neungdong-ro, Gwangjin- \\ gu, Seoul 05030, Korea \\ Tel. +82-2-2030-5010, Fax. +82-2-2030-5029 \\ E-mail:20050101@kuh.ac.kr
}

chronic viral liver disease, and a leading etiology of liver cirrhosis (LC), and hepatocellular carcinoma (HCC) in Korea. ${ }^{1}$ Resection is the optimal treatment option for early-stage HCC; however, the long-term survival is unsatisfactory due to the high recurrence rate of HCC after treatment. ${ }^{2,3}$

The high replicative activity of HBV DNA is associated with high rates of HCC recurrence after curative treatment of patients with chronic hepatitis $\mathrm{B}(\mathrm{CHB})$ diagnosed with 
HCC. Meanwhile, complete viral suppression using potent oral nucleos(t)ide analogues (NAs) such as entecavir (ETV) or tenofovir disoproxil fumarate (TDF) may lower the risk of HCC recurrence and improve the survival of patients undergoing curative treatment for HBV-related HCC. ${ }^{4,5}$ Both TDF and ETV have high genetic barriers and potent antiviral efficacy, and therefore are recommended equally by clinical practice guidelines as first-line antivirals for the management of $\mathrm{CHB}^{6-8}$ However, the relative efficacy of TDF and ETV in the prevention of HCC recurrence after curative treatment is disputed. ${ }^{9,10}$

Hepatic resection is the most appropriate curative option for early HCC. However, many cases of inoperable HCC are attributed to deteriorated liver function or significant portal hypertension. ${ }^{11}$ Thus, to overcome the limitations of resection, loco-regional ablation therapies such as radiofrequency ablation (RFA) have been developed and widely used as alternative therapeutic options for small-sized HCC. ${ }^{12}$ Recently, RFA has been combined with transarterial chemoembolization (TACE) to extend the ablated area for safety margins. ${ }^{13,14}$ Theoretically, RFA is more effective after blocking hepatic arterial blood flow via TACE, because HCC is mainly supplied by hepatic arteries, and several clinical studies demonstrate that this combined therapy reduces local tumor progression around the ablated area of medium-sized HCCs as well as small HCCs. ${ }^{15-17}$ Therefore, the objective of this present study was to retrospectively evaluate the impact on the recurrence and survival of $\mathrm{CHB}$ patients after the combination of TACE and RFA for the treatment of HCC with a curative intent according to the type of NAs.

\section{METHODS}

\section{Ethics statement}

The study protocol was reviewed and approved by the Institutional Review Board of Konkuk University Hospital (IRB no. KUH 1010565). The requirement for informed consent was waived due to the retrospective design of this study.

\section{Patients}

This study was a single-center, retrospective cohort study performed at the Digestive Disease Center, Konkuk University Hospital, Seoul, Korea. The study enrolled 73 CHB patients who underwent the combination treatment of TACE and RFA with curative intent for HCC, and who were treated with TDF or ETV, between January 2007 and December 2019. Patients treated with NAs with low genetic barriers (lamivudine [LAM], adefovir [ADV], or their combination) during the combination treatment with TACE and RFA were excluded in the retrospective study. All enrolled patients were assessed every 3 to 4 months via dynamic CT or MRI. Laboratory parameters such as serum biochemistry, $\alpha$-fetoprotein levels, HBV viral loads were evaluated, followed by a clinical examination during the follow-up period. Recurrence-free survival was defined as time from the commencement of combination of TACE and RFA until HCC recurrence, and overall survival (OS) was defined as time from the initiation of combination treatment of TACE and RFA until death.

Of 73 patients, 30 patients were treated with TDF and 43 patients were treated with ETV. During antiviral therapy, complete viral response (CVR) was defined by HBV-DNA levels less than $24\left(1.38 \log _{10}\right) \mathrm{IU} / \mathrm{mL}$ within 12 months of exposure to NAs. Rapid viral response was arbitrarily defined by HBV-DNA levels less than $24\left(1.38 \log _{10}\right) \mathrm{IU} / \mathrm{mL}$ within 6 months of exposure to NAs. Virological breakthrough was defined by a confirmed increase in HBV DNA level of more than $1 \log _{10} I U / m L$ compared with the nadir HBV DNA level at the time of therapy. Serum HBV DNA levels were assessed using the COBAS Amplicor polymerase chain reaction assay, which has a lower limit of detection of $24\left(1.38 \log _{10}\right) \mathrm{IU} / \mathrm{mL}$ (Roche Molecular Systems, Branchburg, NJ, USA). Antiviral mutations were determined by direct sequencing and multiplex restriction fragment mass polymorphism analysis (GeneMatrix, Seongnam, Korea). The fibrosis-4 (FIB-4) score was measured as a non-invasive marker of liver fibrosis, and calculated using the following formula: age (years) $\times$ aspartate aminotransferase $(\mathrm{IU} / \mathrm{L}) /\left(\right.$ platelet $\left[10^{9} / \mathrm{L}\right] \times$ alanine aminotransferase $\left.[\mathrm{IU} / \mathrm{L}]^{1 / 2}\right){ }^{18}$

Recurrence-free survival (RFS) was defined as the time from date of combination of TACE and RFA to the time of 
recurrence. Patients with no recurrent disease were censored at the last time at which they were known to be recurrence free. Patients who died before experiencing the disease recurrence or lost to follow up were considered censored. The cutoff date for the follow-up visit was 31th May 2021. The Strengthening the Reporting of Observational studies in Epidemiology (STROBE) reporting guidelines were followed (Supplementary Table 1).

\section{Treatment of hepatocellular carcinoma}

Patients initially underwent TACE within 1 month of diagnosis of HCC. TACE was performed in accordance with the standard protocol of our institution. ${ }^{15}$ Briefly, an emulsion consisting 2-10 mL of iodized oil (Lipiodol; Andre Gurbet, Aulnay-sous-Bois, France) and $0.5-1 \mathrm{mg} / \mathrm{kg}$ body weight of doxorubicin hydrochloride (Adriamycin RDF; Ildong Pharmaceutical, Seoul, Korea) was injected slowly under fluoroscopic guidance. Embolization was performed with gelatin-sponge particles (1-2 mm in diameter; Gelfoam; Upjohn, Kalamazoo, MI, USA). Within 5 days after TACE, RFA was performed percutaneously under ultrasonographic or fluoroscopic guidance. RFA was performed according to our institutional standard protocol as previously reported. ${ }^{15} \mathrm{RF}$ application time varied depending on tumor size and imaging feedback; however, it usually ranged between 8 minutes and 12 minutes for single ablation and between 12 minutes and 20 minutes for two overlapping ablations.

\section{Statistical analysis}

Data were mainly expressed as mean with standard deviation. For continuous variables, clinically applicable cut-offs were selected for easy interpretation. Fisher's exact test was used to calculate the statistical significance of the clinical variables. RFS and survival rates were calculated using the Kaplan-Meier method, and differences between groups were compared using the Log-rank test (two groups) or Wilcoxon (Gehan) test (three groups). Multivariate analysis for selecting significant variables was evaluated by Cox proportional hazards models. A $P$-value of less than 0.05 was considered to indicate a significant difference. Statistical analysis was performed with commercially available software (SPSS, version 15.0; SPSS Korea, Seoul, Korea).

Table 1. Baseline clinical characteristics of the patients

\begin{tabular}{|c|c|c|c|}
\hline Parameter & $\begin{array}{l}\text { Patients treated with TDF } \\
\qquad(\mathrm{n}=30)\end{array}$ & $\begin{array}{l}\text { Patients treated with ETV } \\
\qquad(n=43)\end{array}$ & $P$-value \\
\hline Age (years) & $56.0 \pm 7.6$ & $59.4 \pm 8.9$ & 0.090 \\
\hline Sex (male / female) & $22 / 8$ & $31 / 12$ & 0.907 \\
\hline Hepatitis without LC/CP class-A LC/CP class-B LC & $2 / 25 / 3$ & $2 / 38 / 3$ & 0.827 \\
\hline FIB-4 & $4.99 \pm 3.88$ & $5.17 \pm 4.62$ & 0.855 \\
\hline Size of tumor $(\mathrm{cm})$ & $2.0 \pm 0.6$ & $2.2 \pm 0.8$ & 0.174 \\
\hline Number of tumor $(1 / 2 / 3)$ & $25 / 5 / 0$ & $32 / 10 / 1$ & 0.365 \\
\hline Modified UICC stage (I/ II) & $13 / 17$ & $16 / 27$ & 0.599 \\
\hline $\operatorname{AFP}(\mathrm{ng} / \mathrm{mL})$ & $54 \pm 106$ & $91 \pm 236$ & 0.428 \\
\hline Initial HBV-DNA (log10 IU/mL) prior to TDF or ETV & $6.07 \pm 1.01$ & $5.78 \pm 1.32$ & 0.319 \\
\hline HBeAg (positive/ negative) & $16 / 14$ & $19 / 24$ & 0.441 \\
\hline HBV-DNA $\left(\log _{10} \mathrm{IU} / \mathrm{mL}\right)$ at the diagnosis of HCC ${ }^{*}$ & $6.18 \pm 0.81$ & $5.94 \pm 1.11$ & 0.372 \\
\hline
\end{tabular}

Values are presented as mean \pm standard deviations unless otherwise indicated.

TDF, tenofovir disoproxil fumarat; ETV, entecavir; LC, liver cirrhosis; CP, Child-Pugh; FIB-4, the fibrosis-4 score; UICC, Union for International Cancer Control; AFP, alpha fetoprotein; HBV, hepatitis B virus; HBeAg, hepatitis B e antigen; HCC, hepatocellular carcinoma.

*HBV-DNA levels were measured in patients whose HBV-DNA levels were detectable (23 patients treated with TDF vs. 33 patients treated with ETV). 


\section{RESULTS}

\section{Baseline characteristics of patients}

The patients' characteristics are summarized in Table 1. The 73 enrolled patients included 53 men and 20 women, with a mean age of $58.0 \pm 8.5$ years at the time of combination treatment of TACE and RFA. Fifty-seven patients had a single HCC nodule, and 16 had two to three nodules. According to the modified Union for International Cancer Control staging system, 29 and 44 patients were diagnosed with stage I and stage II HCC, respectively. The median maximum tumor diameter was $2.2 \mathrm{~cm}$ (range, $1-4.8 \mathrm{~cm}$ ). Five patients manifested $\mathrm{CHB}$ without LC; 62 patients were diagnosed with Child-Pugh class A LC, and 6 patients had Child-Pugh class B LC.

Thirty patients were treated with TDF, and 43 patients were treated with ETV during the combination treatment with TACE and RFA. Prior to TDF or ETV medication, initial viral loads were 5.9 (range, 2.8-8.1) $\log _{10}$ IU/L. Thirtyfive patients tested positive for hepatitis B e antigen ( $\mathrm{HBeAg}$ ). Ten of 30 patients treated with TDF experienced suboptimal response or viral breakthrough to LAM, ADV, ETV, or their combination prior to TDF therapy. Among 43 patients treat-

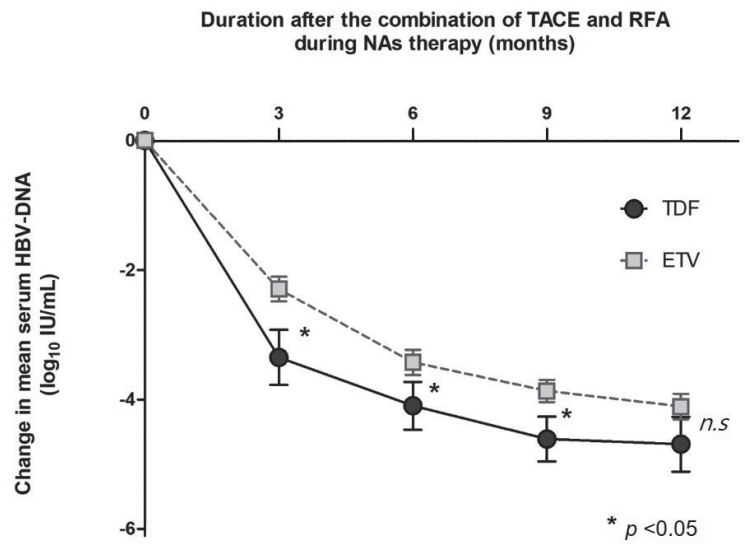

Figure 1. Changes in mean log values of the serum hepatitis $B$ virus (HBV)-DNA levels from the date of combination treatment of transarterial chemoembolization (TACE) and radiofrequency ablation (RFA) during nucleos(t)ide analogue (NA) therapy. The decrease in HBV-DNA was significant in patients treated with tenofovir disoproxil fumarate (TDF) than in patients treated with entecavir (ETV) at 3, 6, and 9 months after the combination treatment of TACE and RFA $(P<0.05)$. ed with ETV, 11 showed suboptimal response or viral breakthrough to LAM, ADV, or their combination prior to ETV therapy. Among 30 patients treated with TDF, 16 patients were treatment-naïve, and 7 patients achieved CVR at the di agnosis of HCC. Among 43 patients treated with ETV, 18 patients were treatment-naÏve, and 10 patients achieved CVR at the diagnosis of HCC. At the diagnosis of HCC (the date of combination of TACE and RFA), HBV-DNA levels of 23 patients treated with TDF and 33 patients treated with ETV were detectable $\left(>1.38 \log _{10} \mathrm{IU} / \mathrm{mL}\right)$.

\section{Antiviral responsiveness}

Fig. 1 shows the mean changes in the HBV-DNA level from the date of the combination of TACE and RFA to each point. The patients whose HBV-DNA levels were undetectable at the diagnosis of HCC were excluded. The decrease in HBV-DNA was significantly more prominent in patients treated with TDF than those with ETV at 3, 6, and 9 months from the date of combination treatment of TACE and RFA $(P<0.05)$. Twenty-two of 23 patients treated with TDF were complete viral responders, whereas 23 of 33 patients showed complete responsiveness to ETV at 1 year after the date of combination of TACE and RFA $(P=0.016)$.

\section{Recurrence and survival}

During a follow-up of $73 \pm 34$ months, 51 experienced recurrence of HCC. The estimated 1- , 3-, and 5-year RFS rates were $86.3 \%, 53.4 \%$, and $36.7 \%$, respectively. Among potential variables, such as sex, age, presence of LC, Child-Pugh class B (vs. class A or non-LC), FIB-4, tumor size, tumor number, HBeAg, antiviral resistance, or type of NAs, the tumor size (hazard ratio [HR], 1.590; 95\% confidence-interval [CI], 1.106-2.285; $P=0.012$ ) and Child-Pugh class B (vs. class A LC or non-LC; HR, 5.794; 95\% CI, 2.311-14.523; $P=0.001$ ) were significantly associated with HCC recurrence in univariate and multivariate analyses (Table 2). Meanwhile, the type of NAs (TDF versus ETV) was not statistically associated with recurrence of HCC after treatment with the combination of TACE and RFA (Fig. 2).

At the end of follow-up, 14 patients were dead due to complications of portal hypertension $(n=2)$, and progression of 
recurrent HCC $(n=12)$. The estimated 1-, 3-, and 5-year OS rates were $98.6 \%, 94.4 \%$, and $83.3 \%$, respectively. Univariate and multivariate analyses indicated that Child-Pugh class B

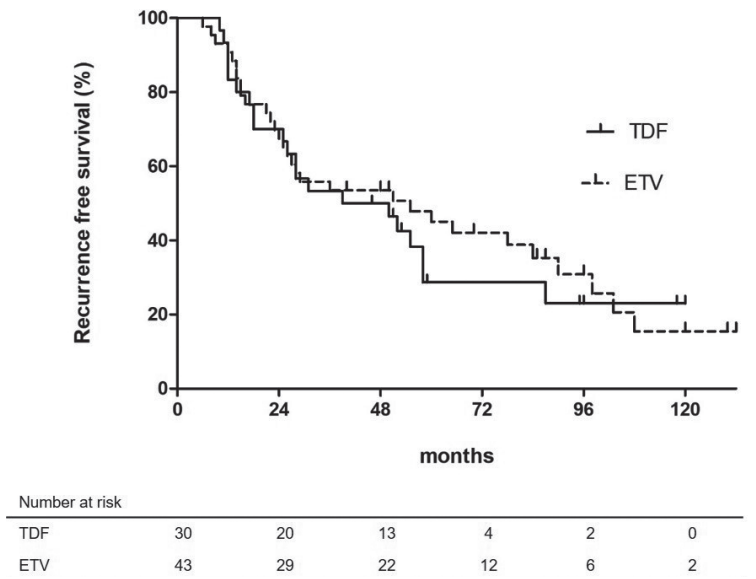

Figure 2. Cumulative recurrence-free survival after combination therapy with transarterial chemoembolization and radiofrequency ablation is shown. Kaplan-Meier curves reveal no significant difference in recurrence-free survival among patients treated with tenofovir disoproxil fumarate (TDF) compared with entecavir (ETV). (vs. class A LC or non-LC; HR, 7.357; 95\% CI, 2.100-25.777; $P=0.002$ ) was the only independent unfavorable prognostic factor for OS (Table 2). Meanwhile, type of NAs (TDF versus



Figure 3. Cumulative overall survival after combination therapy with transarterial chemoembolization and radiofrequency ablation is shown. Kaplan-Meier curves reveal no significant difference in overall survival among patients treated with tenofovir disoproxil fumarate (TDF) compared with entecavir (ETV).

Table 2. Univariate and multivariate analyses of prognostic factors for recurrence-free survival and overall survival

\begin{tabular}{|c|c|c|c|c|c|c|c|c|}
\hline \multirow{3}{*}{ Factor } & \multicolumn{4}{|c|}{ Recurrence-free survival } & \multicolumn{4}{|c|}{ Overall survival } \\
\hline & \multirow{2}{*}{$\begin{array}{c}\text { Univariate } \\
P \text {-value }\end{array}$} & \multicolumn{3}{|c|}{ Multivariate } & \multirow{2}{*}{$\frac{\text { Univariate }}{P \text {-value }}$} & \multicolumn{3}{|c|}{ Multivariate } \\
\hline & & HR & $95 \% \mathrm{Cl}$ & $P$-value & & HR & $95 \% \mathrm{Cl}$ & $P$-value \\
\hline Age (years) & 0.707 & & & & 0.273 & & & \\
\hline Sex (male) & 0.867 & & & & 0.983 & & & \\
\hline LC (+) & 0.203 & & & & 0.279 & & & \\
\hline CP class B & 0.001 & 5.794 & $2.311-14.523$ & 0.001 & $<0.001$ & 7.357 & $2.100-25.777$ & 0.002 \\
\hline FIB-4 & 0.363 & & & & 0.128 & & & \\
\hline Size of tumor (cm) & 0.011 & 1.590 & $1.106-2.285$ & 0.012 & 0.388 & & & \\
\hline Number of tumor & 0.273 & & & & 0.359 & & & \\
\hline Modified UICC stage II & 0.077 & & & & 0.384 & 1.197 & $0.351-4.082$ & 0.774 \\
\hline $\operatorname{AFP}(\mathrm{ng} / \mathrm{mL})$ & 0.365 & & & & 0.550 & & & \\
\hline Initial HBV-DNA ( $\left.\log _{10} \mathrm{IU} / \mathrm{mL}\right)$ & 0.675 & & & & 0.993 & & & \\
\hline $\mathrm{HBeAg}(+)$ & 0.369 & & & & 0.250 & & & \\
\hline $\begin{array}{l}\text { Initial HBV-DNA }\left(\log _{10} \mid \mathrm{U} / \mathrm{mL}\right) \\
\text { at diagnosis of HCC }\end{array}$ & 0.372 & & & & 0.665 & & & \\
\hline Presence of antiviral mutants & 0.297 & & & & 0.766 & & & \\
\hline TDF therapy (vs. ETV therapy) & 0.740 & 1.409 & $0.773-2.565$ & 0.263 & 0.919 & 0.860 & $0.280-2.642$ & 0.792 \\
\hline
\end{tabular}

HR, hazard ratio; Cl, confidence interval; LC, liver cirrhosis; CP class, Child-Pugh classification; FIB-4, the fibrosis-4 score; UICC, Union for International Cancer Control; AFP, alpha fetoprotein; HBV, hepatitis B virus; HBeAg, hepatitis B e antigen; HCC, hepatocellular carcinoma; TDF, tenofovir disoproxil fumarate; ETV, entecavir. 
ETV) was not significantly associated with patients' survival (Fig. 3).

\section{DISCUSSION}

The use of NAs has been recommended to reduce the risk of recurrence and mortality after surgical resection of HBVrelated HCC. However, the effect of NA therapies on the prognosis of HBV-related HCC patients after combined treatment with TACE is not clearly established. In this retrospective study, we evaluated clinical outcomes of HCC recurrence and OS after treatment with a combination of TACE and RFA with curative intent depending on the use of highpotency NAs such as TDF or ETV.

The present study enrolled the patients who underwent combination of TACE and RFA, instead RFA alone, to minimize the influence of local tumor progression. Several studies have shown that combination of TACE and RFA significantly improved local tumor control, and overall survival rates compared with TACE or RFA alone..$^{19-21}$ In the present study, the recurrence of HCC was observed in more than twothirds of patients who were exposed to a combination therapy of TACE+RFA, comparable to previously reported rates. ${ }^{19-21}$ Multivariate analysis showed that $\mathrm{CP}$ class $\mathrm{B}$ cirrhosis was identified as significant independent factor of HCC recurrence. Thus, improvement in the liver functional reserve and degree of cirrhosis might directly or indirectly attenuate the risk of HCC recurrence, suggesting the need for concomitant medical treatment such as highly potent NAs for HBV related-HCC to enhance liver functional reserve or degree of LC. From a prognostic perspective, the mortality rate was $19.2 \%$, with 14 deaths during a median follow-up of 73 months. Most of the patients (12/14) died because of recurrent HCC progression, and two out of 14 died due to complications associated with portal hypertension. The patient's prognosis might be explained by the complex relationship between HCC and underlying LC. In the present study, predictably, multivariate analysis showed that severity of cirrhosis was a significant factor associated with patient's prognosis. Therefore, not only HCC recurrence but also underlying liver functional reserve directly affected the patients' survival.

During NAs therapy, the decrease in HBV-DNA was significantly more prominent in patients treated with TDF than those exposed to ETV at 3, 6, and 9, months after combination treatment of TACE and RFA. Twenty-two of 23 patients treated with TDF were complete responders, whereas 23 of 33 patients treated with ETV were complete responders at 1 year after the combination of TACE and RFA $(P=0.016)$. Thus, these data suggest that TDF is superior to ETV for achieving a CVR. The study enrolled a substantial number of patients who experienced suboptimal response or viral breakthrough to LAM, ADV or their combination prior to TDF or ETV therapy, and HBV strains resistant to low genetic barriers (LAM, ADV, or their combination) reduce the susceptibility to ETV therapy, whereas those HBV strains are effectively suppressed by TDF therapy. ${ }^{22,23}$ Therefore, TDF is the better treatment option due to its higher barrier to antiviral resistance, especially in patients with HBV-related HCC manifesting suboptimal response or viral breakthrough to low genetic barriers. Nonetheless, this study demonstrates that the type of antiviral treatment (TDF versus ETV) did not significantly affect the patients' prognosis. The risks of HCC recurrence and death were not statistically different between those treated with TDF and ETV in this retrospective study. Previous studies have consistently showed that the highly potent NAs decreased the risk of HCC recurrence after curative treatment such as resection or RFA. A nationwide cohort study from Taiwan revealed that the risk of HCC recurrence was lower in patients treated with NAs and NA therapy was independently related to decreased HCC recurrence. ${ }^{4}$ A meta-analysis also showed that the beneficial effects of NAs were consistent with reduced HCC recurrence. ${ }^{24}$ Therefore, highly potent NAs are strongly recommended for patients with HBV-related HCC after curative therapy of HCC to reduce the risk of HCC recurrence. However, it is debatable what is the optimal choice of NAs for patients who underwent hepatic resection for the treatment of HBV-related HCC. A recent retrospective large-scale cohort study by Choi et al. ${ }^{10}$ demonstrated that TDF was associated with a decreased risk of HCC recurrence than ETV after surgical resection of HCC. Their clinical data may be supported by in vitro study 
showing that unlike nucleoside analogues including ETV, nucleotide analogues including TDF might exhibit an additional immunomodulatory effect to induce favorable immune response for HBV elimination. ${ }^{25}$ However, a subsequent study by Lee et al. ${ }^{9}$ showed conflicting results that HCC recurrence after curative treatment was not significantly different between ETV and TDF. They suggest that favorable outcomes with TDF compared with ETV reported by Choi et al. ${ }^{10}$ might be related to a selection bias due to the gap between approval times of ETV and TDF. Our data also revealed no difference in the rates of HCC recurrence and all-cause mortality between patients treated with TDF versus ETV even though TDF is more effective than ETV to achieve rapid viral suppression. Therefore, our data suggest that, compared with the degree of LC or tumoral factors, NA type (TDF versus ETV) might have a relatively lower impact on the prognosis in terms of HCC recurrence and mortality in patients exposed to TACE combined with RFA with curative intent for HBV-related HCC.

This study has several important limitations. First, this was a retrospective study with all its inherent limitations. In the absence of a control group, the therapeutic efficacy of combination therapy could not be validated. Second, the number of patients was relatively small, including mainly HBV population diagnosed in a single tertiary institution, making it difficult to extrapolate these results. Finally, the majority of HCC tumors were not confirmed histopathologically, but were considered HCC based on clinical criteria. However, we think this was an insurmountable limitation in real clinical practice of HCC management.

In conclusion, the retrospective study demonstrates that TDF is superior to ETV for achieving CVR in patients with CHB who underwent the combined treatment of TACE and RFA for HBV-related HCC. However, the prognoses in terms of recurrence and mortality were not significantly different in patients treated with TDF compared with ETV.

\section{Conflicts of Interest}

Won Hyeok Choe has served on the Editorial Board of J Liver Cancer since July 2020. He was not involved in the review process of this article. Otherwise, the authors have no conflicts of interest to disclose.

\section{Ethics Statement}

Based on the Declaration of Helsinki, the Institutional Review Board of Konkuk University Hospital approved the retrospective use of the clinical, biochemical, and radiographic data for the present study (IRB no. KUH 1010565). The requirement for informed consent was waived due to the retrospective design of this study.

\section{Funding Statement}

No funding to declare.

\section{Data Availability}

The datasets generated or analyzed during the study are available from the corresponding author on reasonable request.

\section{ORCID}

Jae Min Park https://orcid.org/0000-0001-9045-6543

Won Hyeok Choe Jeong Han Kim https://orcid.org/0000-0002-8019-5412

So Young Kwon https://orcid.org/0000-0002-8383-8524

Byung Chul Yoo https://orcid.org/0000-0003-4290-1950 https://orcid.org/0000-0001-9726-9492

\section{Author Contribution}

Study concept and design: JMP, WHC, JHK, SYK, BCY

Data acquisition, analysis, and interpretation of the data: JMP, WHC, JHK, SYK, BCY

Drafting of manuscript: JMP, WHC, JHK, SYK

Statistical analysis: JMP

All authors have reviewed and approved the final version of the manuscript.

\section{Supplementary Material}

Supplementary data can be found with this article online http://www.e-jlc.org/html/https://doi.org/10.17998/jlc.2021.09.22.

\section{References}

1. Liang LY, Wong GL. Unmet need in chronic hepatitis B manage- 
ment. Clin Mol Hepatol 2019;25:172-180.

2. Schweitzer A, Horn J, Mikolajczyk RT, Krause G, Ott JJ. Estimations of worldwide prevalence of chronic hepatitis $B$ virus infection: a systematic review of data published between 1965 and 2013. Lancet 2015;386:1546-1555.

3. Kim BK, Park JY, Kim DY, Kim JK, Kim KS, Choi JS, et al. Persistent hepatitis B viral replication affects recurrence of hepatocellular carcinoma after curative resection. Liver Int 2008;28:393-401.

4. Wu CY, Chen YJ, Ho HJ, Hsu YC, Kuo KN, Wu MS, et al. Association between nucleoside analogues and risk of hepatitis $B$ virusrelated hepatocellular carcinoma recurrence following liver resection. JAMA 2012;308:1906-1914.

5. Yin J, Li N, Han Y, Xue J, Deng Y, Shi J, et al. Effect of antiviral treatment with nucleotide/nucleoside analogs on postoperative prognosis of hepatitis B virus-related hepatocellular carcinoma: a two-stage longitudinal clinical study. J Clin Oncol 2013;31:36473655.

6. European Association for the Study of the Liver. EASL 2017 Clinical practice guidelines on the management of hepatitis B virus infection. J Hepatol 2017;67:370-398.

7. Sarin SK, Kumar M, Lau GK, Abbas Z, Chan HL, Chen CJ, et al. Asian-Pacific clinical practice guidelines on the management of hepatitis B: a 2015 update. Hepatol Int 2016;10:1-98.

8. Terrault NA, Lok ASF, McMahon BJ, Chang KM, Hwang JP, Jonas $M M$, et al. Update on prevention, diagnosis, and treatment of chronic hepatitis B: AASLD 2018 hepatitis B guidance. Hepatology 2018;67:1560-1599.

9. Lee JH, Kim BK, Park SY, Tak WY, Park JY, Kim DY, et al. The efficacies of entecavir and tenofovir in terms of enhancing prognosis after curative treatment of hepatitis B virus-related hepatocellular carcinoma. Eur J Intern Med 2021;89:48-55.

10. Choi J, Jo C, Lim YS. Tenofovir versus entecavir on recurrence of hepatitis $B$ virus-related hepatocellular carcinoma after surgical resection. Hepatology 2021;73:661-673.

11. Villanueva A. Hepatocellular carcinoma. N Engl J Med 2019;380:1450-1462.

12. Kim YS, Lim HK, Rhim H, Lee MW, Choi D, Lee WJ, et al. Ten-year outcomes of percutaneous radiofrequency ablation as first-line therapy of early hepatocellular carcinoma: analysis of prognostic factors. J Hepatol 2013;58:89-97.

13. Kim JW, Kim JH, Won HJ, Shin YM, Yoon HK, Sung KB, et al. Hepatocellular carcinomas $2-3 \mathrm{~cm}$ in diameter: transarterial chemoembolization plus radiofrequency ablation vs. radiofrequency ablation alone. Eur J Radiol 2012;81:e189-e193.

14. Morimoto M, Numata K, Kondou M, Nozaki A, Morita S, Tanaka K. Midterm outcomes in patients with intermediate-sized hepatocellular carcinoma: a randomized controlled trial for determining the efficacy of radiofrequency ablation combined with transcatheter arterial chemoembolization. Cancer 2010;116:5452-5460.

15. Choe WH, Kim YJ, Park HS, Park SW, Kim JH, Kwon SY. Shortterm interval combined chemoembolization and radiofrequency ablation for hepatocellular carcinoma. World J Gastroenterol 2014;20:12588-12594.

16. Kang SG, Yoon CJ, Jeong SH, Kim JW, Lee SH, Lee KH, et al. Single-session combined therapy with chemoembolization and radiofrequency ablation in hepatocellular carcinoma less than or equal to $5 \mathrm{~cm}$ : a preliminary study. J Vasc Interv Radiol 2009;20:15701577.

17. Li W, Ni CF. Current status of the combination therapy of transarterial chemoembolization and local ablation for hepatocellular carcinoma. Abdom Radiol (NY) 2019;44:2268-2275.

18. Xiao G, Yang J, Yan L. Comparison of diagnostic accuracy of aspartate aminotransferase to platelet ratio index and fibrosis- 4 index for detecting liver fibrosis in adult patients with chronic hepatitis $B$ virus infection: a systemic review and meta-analysis. Hepatology 2015;61:292-302.

19. Kim AR, Park E, Kwon SY, Park SJ, Kim YJ, Yoo BC, et al. Efficacy and safety of combined radiofrequency ablation with transarterial chemoembolization in patients with Barcelona Clinic Liver Cancer Stage A hepatocellular carcinoma ineligible for curative treatment. Korean J Gastroenterol 2019;73:167-176.

20. Fujimori M, Takaki H, Nakatsuka A, Uraki J, Yamanaka T, Hasegawa $T$, et al. Survival with up to 10 -year follow-up after combination therapy of chemoembolization and radiofrequency ablation for the treatment of hepatocellular carcinoma: single-center experience. J Vasc Interv Radiol 2013;24:655-666.

21. Kim JW, Kim JH, Sung KB, Ko HK, Shin JH, Kim PN, et al. Transarterial chemoembolization vs. radiofrequency ablation for the treatment of single hepatocellular carcinoma $2 \mathrm{~cm}$ or smaller. Am J Gastroenterol 2014;109:1234-1240.

22. Lim YS. Management of antiviral resistance in chronic hepatitis $B$. Gut Liver 2017;11:189-195.

23. Ghany MG, Doo EC. Antiviral resistance and hepatitis B therapy. Hepatology 2009;49(5 Suppl):S174-84.

24. Wong JS, Wong GL, Tsoi KK, Wong VW, Cheung SY, Chong CN, et al. Meta-analysis: the efficacy of anti-viral therapy in prevention of recurrence after curative treatment of chronic hepatitis B-related hepatocellular carcinoma. Aliment Pharmacol Ther 2011;33:11041112.

25. Murata K, Tsukuda S, Suizu F, Kimura A, Sugiyama M, Watashi $K$, et al. Immunomodulatory mechanism of acyclic nucleoside phosphates in treatment of hepatitis B virus infection. Hepatology 2020;71:1533-1545. 